\title{
Good agreement between echocardiography and impedance cardiography in the assessment of left ventricular performance in hypertensive patients
}

\author{
Rodrigo Nazário Leão, Pedro Marques Da Silva, Rita Marques Pocinho, Marta \\ Alves, Daniel Virella \& Roberto Palma Reis
}

To cite this article: Rodrigo Nazário Leão, Pedro Marques Da Silva, Rita Marques Pocinho, Marta Alves, Daniel Virella \& Roberto Palma Reis (2017): Good agreement between echocardiography and impedance cardiography in the assessment of left ventricular performance in hypertensive patients, Clinical and Experimental Hypertension, DOI: 10.1080/10641963.2017.1392558

To link to this article: https://doi.org/10.1080/10641963.2017.1392558

Published online: 27 Nov 2017.

Submit your article to this journal $\llbracket$

Џ Article views: 18

View related articles

View Crossmark data $\asymp$ 


\title{
Good agreement between echocardiography and impedance cardiography in the assessment of left ventricular performance in hypertensive patients
}

\author{
Rodrigo Nazário Leão (10), Pedro Marques Da Silva ${ }^{\mathrm{b}, \mathrm{c}}$, Rita Marques Pocinho ${ }^{\mathrm{a}}$, Marta Alves ${ }^{\mathrm{d}}$, Daniel Virella ${ }^{\mathrm{f}}$, \\ and Roberto Palma Reis ${ }^{\mathrm{b}, \mathrm{e}}$ \\ aUnidade Funcional Medicina 2, Hospital São José, Centro Hospitalar Lisboa Central-EPE, Lisboa, Portugal; bNOVA Medical School, Universidade \\ NOVA de Lisboa, Lisboa, Portugal; 'Núcleo de Investigação Arterial, Unidade Funcional Medicina 4, Hospital Santa Marta, Centro Hospitalar Lisboa

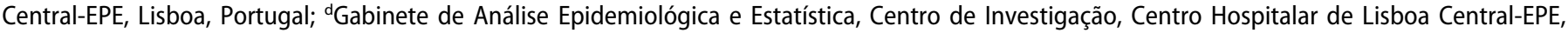 \\ Lisboa, Portugal; eUnidade de Cardiologia, Hospital Pulido Valente, Centro Hospitalar Lisboa Norte-EPE, Lisboa, Portugal; ${ }^{\mathrm{f} G a b i n e t e ~ d e ~ A n a ́ l i s e ~}$ \\ Epidemiológica e Estatística, Centro de Investigação, Centro Hospitalar Lisboa Central-EPE, Lisboa, Portugal
}

\begin{abstract}
Background: Impedance cardiography (ICG) is a noninvasive hemodynamic monitoring tool which can define hypertensive patients' hemodynamic profiles and help to tailor antihypertensive therapy. This study assesses the concordance between ICG-derived indexes used to evaluate left ventricular performance and transthoracic echocardiography (TTE) in hypertensive patients. Methods: In this IMPEDDANS post-hoc analysis, the ICG-derived indexes are compared with TTE by Bland-Altman method. Statistical significance of the relationship between the values obtained was assessed by generalized linear mixed-effects models. Results: In supine position, Bland-Altman analysis showed good concordance for cardiac output (CO) (mean difference of $0.006 \mathrm{~mL} / \mathrm{min}[-0.120 ; 0.133]$ ), cardiac index $(\mathrm{Cl})$ (mean difference of $0.016 \mathrm{~mL} / \mathrm{min} / \mathrm{m}^{2}[-0.471 ; 0.504]$ ), pre-ejection period (PEP) (mean difference of $-0.216 \mathrm{~ms}[-4.510 ; 4.077]$ ), left ventricular ejection time (LVET) (mean difference of $-0.140 \mathrm{~ms}[-6.573 ; 6.293]$ ), and systolic time ratio (STR) (mean difference of $-0.00004[-0.008$; $0.008]$ ). In orthostatic position, good concordance was found for CO (mean difference $0.028 \mathrm{~mL}$ / $\min [-2.036 ; 1.980]$ ), $\mathrm{Cl}$ (mean difference $-0.012 \mathrm{~mL} / \mathrm{min} / \mathrm{m}^{2}[-1.063 ; 1.039]$ ), and STR (mean difference $-0.101[0.296 ; 0.094])$. No significant difference between methods was identified by the linear mixed-effects models. Conclusion: The ICG-derived indexes CO, CI, PEP, LVET, and STR in supine position have good agreement with TTE. Therefore, ICG can be used to accurately evaluate left ventricular performance.
\end{abstract}

\section{ARTICLE HISTORY}

Received 31 August 2017

Revised 21 September 2017

Accepted 27 September

2017

\section{KEYWORDS}

Echocardiography; impedance cardiography; hypertension; left ventricular performance; systolic time intervals

\section{Background}

Impedance cardiography (ICG) has evolved in recent years, it is already used in several setting (e.g., evaluation of postural cardiac rehabilitation, pacemaker optimization, sleep study, hemodynamic monitoring in pregnancy and in the outpatient setting), and it has become an attractive and economical alternative to the clinical approach to hypertensive patients (1-7). ICG is a noninvasive hemodynamic monitoring tool, nonoperator dependent and non-expensive, which can characterize hemodynamic profiles and contribute to optimize antihypertensive therapy $(8,9)$. The use of ICG hemodynamic parameters has been proposed to guide pharmacologic prescription according to the pathophysiological mechanism involved in the sustained elevation of blood pressure (BP) (10-15). This personalized, guided hemodynamic therapy approach reveals both efficacy and cost-effectivity (10-15).

The evolution of hypertensive heart disease toward heart failure (HF) involves structural and geometry modifications which can impair left ventricular (LV) function $(9,16)$. Recent reports suggest that the ICG-derived systolic time intervals may be used to monitor impaired systolic and/or diastolic LV function; however, there are conflicting results concerning the screening of LV impairment and in the validation of the ICGderived indexes. Therefore, it is necessary to validate the method in specific clinical settings (17-25). This study verifies the agreement between ICG-derived indexes used in evaluating LV function and the clinical standard, in patients with severe hypertension (HT).

\section{Methods}

\section{Study design}

In this post-hoc analysis of data from the IMPEDDANS project (Impedance Cardiography in the Evaluation of Left Ventricular Diastolic dysfunction in Patients with Arterial Hypertension Study), the ICG-derived indexes are compared with contemporary transthoracic echocardiography (TTE)derived indexes, used nowadays as the clinical standard. The cardiac output (CO), cardiac index (CI), pre-ejection period 
(PEP), left ventricular ejection time (LVET), and systolic time ratio (STR) are assessed by ICG (in supine and orthostatic positions) and TTE. The project protocol was approved by the hospital's Ethics Committee (approval number 166/2014). Informed consent was obtained from each patient. IMPEDDANS is registered on ClinicalTrials.gov (ID: NCT03209141).

\section{Sampling}

Patients of either gender, aged 18-75 years, with grade 2 or 3 HT (systolic BP $\geq 160 \mathrm{mmHg}$ and/or diastolic $\mathrm{BP} \geq 100 \mathrm{mmHg}$ ) and/or with resistant $\mathrm{HT}$ (according the definition of ESH/ESC guidelines) (26), followed up in ambulatory clinic of an Internal Medicine Department of a tertiary referral hospital were eligible for recruitment.

Patients were excluded in the presence of pregnancy, height $<120 \mathrm{~cm}$ or $>230 \mathrm{~cm}$, weight $<30 \mathrm{~kg}$ or $>155 \mathrm{~kg}$, HF II-IV NYHA (27), heart rate (HR) $<50 \mathrm{bpm}$ or $>110 \mathrm{bpm}$, atrial fibrillation or flutter, more than three premature ventricular contractions per hour, complete left bundle branch or atrioventricular block, pacemaker, severe valvulopathy, constrictive pericarditis, hypertrophic and restrictive cardiomyopathy, ischemic heart disease and/or segmental kinetics anomalies assessed by echocardiography, left ejection fraction $<50 \%$, or poor echocardiographic window. Recruitment began in January 2015 and was finished in July 2017.

\section{Evaluations and data collection}

Participants were invited to be systematically assessed by ICG and echocardiography, with a maximum interval of 8 days in between. To ensure that both tests were performed under similar conditions, evaluations matching variations $>10 \%$ in $\mathrm{BP}$ or variations $>5 \%$ in $\mathrm{HR}$ were not considered. These patients were asked to repeat the tests. If the variations persisted, they were excluded.

ICG was carried out by an experienced cardiopneumology technician with Niccomo - Non-Invasive Continuous Cardiac Output Monitor (Medis GmbH, Ilmenau, Germany) according the approved department protocol. Patients were required to have $6 \mathrm{~h}$ of fasting but took their usual antihypertensive drugs. With patients in dorsal decubitus, it was carried out in supine position during 20 min (continuous recording) and $70^{\circ}$ orthostatism on a tilting table (10 min in continuous recording). The test was interrupted whenever occurred syncope or pre-syncope; dizziness, nausea, and malaise associated with poorly tolerated hypotension and/or bradycardia; pain/precordial discomfort; EKG ST segment changes; systolic BP $>210 \mathrm{mmHg}$.

TTE was performed in Vivid E9 and S5 devices (GE Healthcare, Chicago, IL, USA) always by experienced cardiologists. To ensure uniformity of evaluation and correct evaluation, all exams were reviewed by another independent cardiologist. The exam was performed with the patients in left lateral decubitus. $\mathrm{CO}$ was calculated based on measurement of the flow velocity time integral (VTI) measured over the aortic valve (AoV), measured from an apical four-chamber view with angle correction, if necessary, multiplied by the

area of the AoV:CO $=\mathrm{AoV}$ area $\times \mathrm{VTI} \times \mathrm{HR}$, where the $\mathrm{AoV}$ diameter was determined by triplicate measurements of the internal diameter of the AoV hinge points: AoV area $=(0.5 \times \text { diameter })^{2} \times 3.14(28)$. LVET $(\mathrm{ms})$ was measured as the duration of flow using standard pulsed wave Doppler with sample volume in the LV outflow tract just below the AoV leaflets. PEP (ms) was measured as the time interval between R wave on ECG and beginning of LV outflow tract flow. The ratio of PEP to LVET was also obtained (29).

The correlation between the parameters obtained by ICG (both in supine and orthostatic positions) and TTE was assessed by Spearman correlation test; the strength of correlation was interpreted according to Hinkle et al (30). To evaluate the agreement between ICG (both in supine and orthostatic positions) and TTE for measuring CO, CI, PEP, LVET, and STR, Bland-Altman method was used. Additionally, the statistical significance of the relationship between the values obtained by ICG and TTE was assessed using generalized linear mixed-effects models. The level of significance $\alpha=0.05$ was considered. Data were analyzed with Stata 11.0 (StataCorp. 2011, Stata Statistical Software: Release 12. College Station, TX: StataCorp LP).

\section{Results}

IMPEDDANS study recruited 167 patients. Ten patients were excluded: three had syncope during ICG, three gave up and did not perform the tests, three had a significant dysrhythmia, and one was diagnosed as having an atrial septal defect. From the 157 hypertensive patients included, 102 (65\%) had LV diastolic dysfunction (diagnosed by echocardiogram). Table 1 shows the main demographic and clinical characteristics of the patients.

The values obtained by ICG (in both supine and orthostatic positions) and by TTE are presented in Table 1. The values of every parameter obtained by ICG in supine position had a very high correlation with their equivalents obtained by TTE; on the other hand, the TTE-derived parameters had low or moderate correlation with the values obtained by ICG in orthostatic position (Table 2 and Supplementary Figure 1).

Table 1. Main characteristics of the effective sample $(n=157)$.

\begin{tabular}{lc}
\hline Males, $n$ (\%) & $88(56.1)$ \\
\hline Age, mean (SD) & $63(10)$ \\
Caucasian, $n$ (\%) & $142(90.4)$ \\
HT duration (months), mean (SD) & $120(104)$ \\
Resistant HT, $n$ (\%) & $117(74.5)$ \\
Obesity, $n$ (\%) & $82(52.2)$ \\
Diabetes mellitus, $n(\%)$ & $81(51.6)$ \\
Dyslipidemia, $n$ (\%) & $133(84.7)$ \\
CKD, $n(\%)$ & $49(31.2)$ \\
Stroke, $n(\%)$ & $36(22.9)$ \\
COPD, $n(\%)$ & $20(12.7)$ \\
OSA, $n(\%)$ & $31(19.7)$ \\
Smoker, $n(\%)$ & $34(21.7)$ \\
Alcoholic, $n$ (\%) & $16(10.8)$ \\
No. of AHT drugs, median (min-max) & $4(1-7)$ \\
\hline AHT: Antihypertensive; CKD: chronic kidney disease; \\
COPD: chronic obstructive pulmonary disease; \\
HT: hypertension.
\end{tabular}




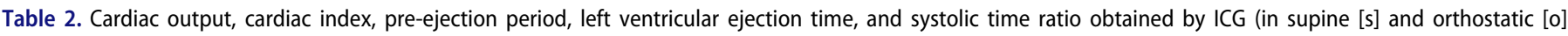
positions) and by transthoracic echocardiography values.

\begin{tabular}{|c|c|c|c|c|c|}
\hline & ICGs & ICGo & TTE & Correlation TTE:ICGs (r) & Correlation TTE:ICGo (r) \\
\hline $\begin{array}{l}\text { CO }(\mathrm{mL} / \mathrm{min}) \text {, median }\left(P_{25}-P_{75}\right) \\
\text { CI }\left(\mathrm{mL} / \mathrm{min} / \mathrm{m}^{2}\right) \text {, median }\left(P_{25}-P_{75}\right) \\
\text { PEP }(\mathrm{ms}) \text {, median }\left(P_{25}-P_{75}\right) \\
\text { LVET }(\mathrm{ms}) \text {, median }\left(P_{25}-P_{75}\right) \\
\text { STR, median }\left(P_{25}-P_{75}\right)\end{array}$ & $\begin{aligned} 5.2 & (4.3-6.0) \\
2.7 & (2.4-3.5) \\
93 & (79-105) \\
332 & (291-369) \\
0.28 & (0.24-0.33)\end{aligned}$ & $\begin{array}{l}5.3(4.5-6.0) \\
2.8(2.5-3.1) \\
117(99-133) \\
307(268-332) \\
0.39(0.32-0.46)\end{array}$ & $\begin{aligned} 5.2 & (4.4-6.0) \\
2.7 & (2.4-3.1) \\
93 & (79-104) \\
330 & (292-369) \\
0.28 & (0.24-0.33)\end{aligned}$ & $\begin{array}{l}0.998 \\
0.996 \\
0.991 \\
0.997 \\
0.996\end{array}$ & $\begin{array}{l}0.577 \\
0.437 \\
0.533 \\
0.491 \\
0.455\end{array}$ \\
\hline
\end{tabular}

$\mathrm{Cl}$ : Cardiac index; CO: cardiac output; LVET: left ventricular ejection time; PEP: pre-ejection period; STR: systolic time ratio; TTE: transthoracic echocardiography. Correlations calculated with Spearman index $(r)$.

The Bland-Altman analysis showed a good concordance between the values of the parameters obtained by TTE and by ICG in supine position (Figure 1(a-e)): for CO, the mean difference was $0.006 \mathrm{~mL} / \mathrm{min}$ (limits of agreement -0.120 $0.133)$; for $\mathrm{CI}$, mean difference $0.016 \mathrm{~mL} / \mathrm{min} / \mathrm{m}^{2}(-0.471-$ $0.504)$; for PEP, mean difference $-0.216 \mathrm{~ms}(-4.510-4.077)$; LVET, mean difference $-0.140 \mathrm{~ms}(-6.573-6.293)$; and STR, mean difference $-0.00004(-0.008-0.008)$.

The Bland-Altman analysis of the values of the parameters obtained by TTE and by ICG in orthostatic position (Figure $1(\mathrm{f}-\mathrm{j})$ ) showed good concordance for CO (mean difference $0.028 \mathrm{~mL} / \mathrm{min}$; limits of agreement -2.036 1.980 ) and CI (mean difference $-0.012 \mathrm{~mL} / \mathrm{min} / \mathrm{m}^{2}$; limits of agreement $-1.063-1.039)$. The agreement was worse for PEP (mean difference $-22.649 \mathrm{~ms}$; limits of agreement -66.316-21.017), LVET (mean difference of $25.617 \mathrm{~ms}$; limits of agreement -73.194-124.429), and STR (mean difference -0.101 ; limits of agreement $-0.296-0.094$ ).

The linear mixed effects models identified no significant difference between the TTE-derived values and those obtained by ICG performed in supine position, in any of the parameters $(p=0.214-0.890)$. For the parameters obtained by ICG performed in orthostatic position, a significant difference to the TTE-derived values was found for PEP, LVET, and STR $(p<0.001)$ (Table 3).

\section{Discussion}

This study found good agreement (Bland-Altman analysis) between the TTE-derived parameters (CO, CI, PEP, LVET, STR) and those ICG-derived evaluated in supine position. The agreement of TTE-derived parameters and those ICG-derived evaluated in orthostatic position was acceptable for $\mathrm{CO}$ and CI but low for PEP, LVET, and STR. These results demonstrate that the ICG in supine position can be used as a reliable and cost-effective alternative for evaluation and monitoring LV performance-derived indexes.

The good agreement found between these two methods may help to reduce doubts about the usefulness of ICG in hypertensive patients. Although there are many published articles about the benefits of ICG and its applicability, there are issues that have conditioned its overall use. Most studies carried out had small samples, the accuracy and reproducibility of each variable was differently determined between trials, and results were conflicting (25). TTE has rarely been used as a reference method to analyze the agreement of the parameters evaluated by ICG and studies presented different results with correlation coefficients ranging from 0.27 to 0.95 $(25,31-35)$.

There are few studies that evaluate the concordance of other parameters besides CO (25). In literature, we find three meta-analysis that group almost all studies that compare the agreement of CO evaluated by ICG with other reference methods. Fuller et al. analyzed 28 studies comparing ICG with the Fick and thermodilution methods and concluded that ICG had a correlation coefficient of 0.81 (36). Raaijmakers et al., in a meta-analysis of 112 studies, determined a correlation coefficient of 0.82 (37). Recently, Peyton and colleagues conducted a new meta-analysis of studies comparing ICG, esophageal Doppler, pulse-wave contour analysis, and the Fick method with thermodilution (38). These authors report a correlation coefficient of 0.79 and low mean difference for ICG revealing better agreement than the other noninvasive methods (38).

Despite these favorable results, some studies have shown a low correlation between ICG and the various methods of hemodynamic evaluation, namely in obese patients, in patients with HF, chronic obstructive pulmonary disease, aortic valvulopathy, dysrhythmia, in septic patients, under mechanical ventilation, post cardiac surgery, among others (39-43). Signal interference, hardware problems that impaired signal sensitivity, or even the incorrect placement of the electrodes are also potential biases in the ICG evaluations (44-46). The technological developments that have been introduced proved to be effective in their resolution, and trials conducted with newer monitors have shown good agreement with other methods $(43,47)$.

Thus, we attribute the good agreement found in this study, with correlation coefficients higher than the global values found in the previous studies $(r=0.99)$ and with low mean differences and agreement limits, to the fact that we excluded factors that influence the evaluations by ICG such as the presence of HF, significant valvulopathy, moderate-to-severe chronic obstructive pulmonary disease, dysrhythmias, among others comorbidities which could bias the evaluations; we included only ambulatory patients; we used a new generation monitor; we performed both examinations according to an well-defined protocol and in a controlled clinical setting, by expert practitioners.

Furthermore, previous studies report good reproducibility of the evaluations performed on the same day but only acceptable reproducibility when performed on different days $(r=0.65-0.86)(48-50)$. The fact that our protocol establishes that the examinations had to be performed in a short period of time (8 days) and that patients could not present variations 

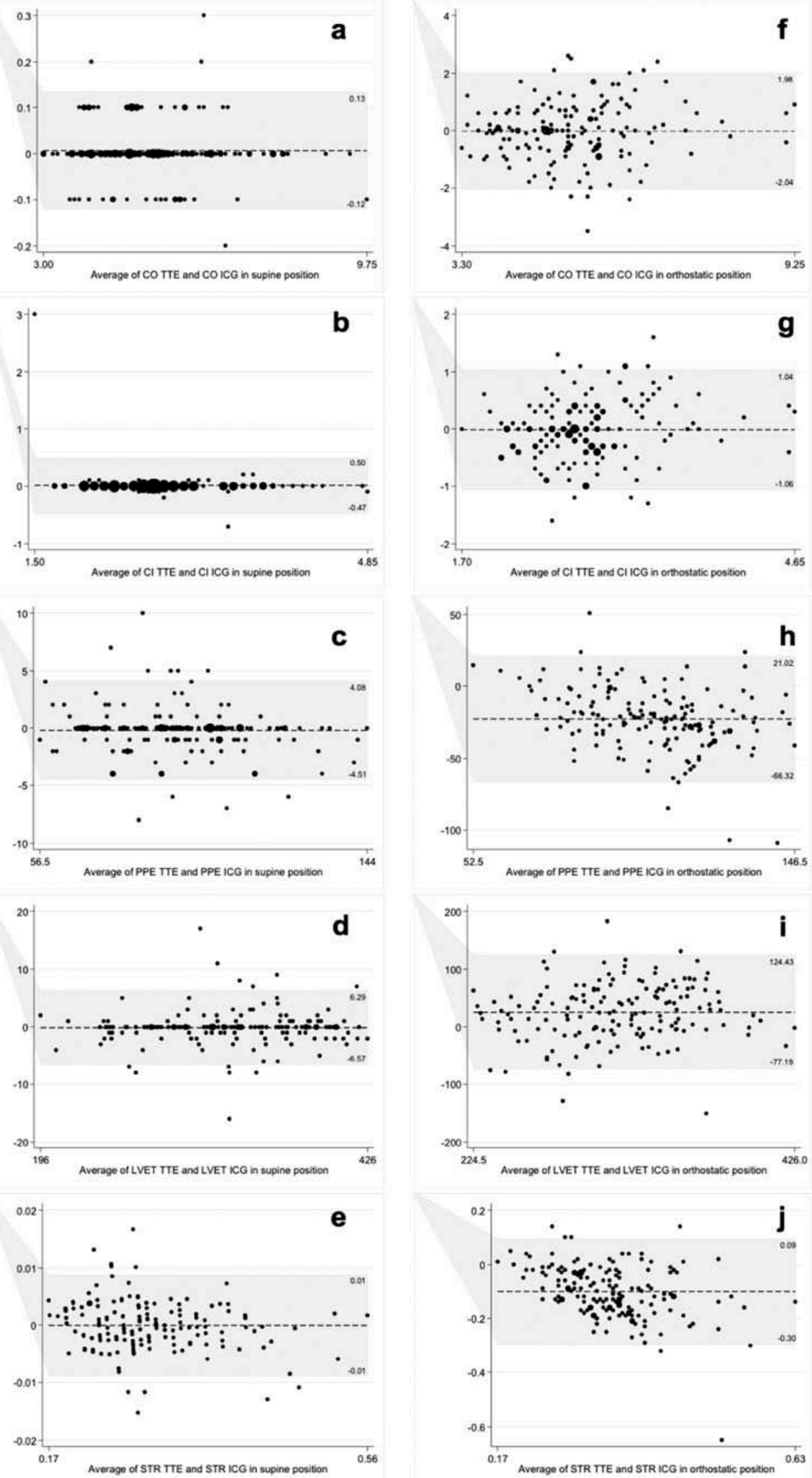

Figure 1. Agreement of the indexes used to evaluate left ventricular performance obtained by TTE and by ICG, both in supine and orthostatic positions. (a) Cardiac output in supine position, (b) cardiac index in supine position, (c) pre-ejection period in supine position, (d) left ventricular ejection time in supine position, (e) systolic time ratio in supine position, (f) cardiac output in orthostatic position, (g) cardiac index in orthostatic position, (h) pre-ejection period in orthostatic position, (i) left ventricular ejection time in orthostatic position, and (j) systolic time ratio in orthostatic position.

Cl: Cardiac index; CO: cardiac output; ICG: impedance cardiography; LVET: left ventricular ejection time; PPE: pre-ejection period; STR: systolic time ratio; TTE: transthoracic echocardiography. 
Table 3. Relationship between the values of the parameters used to evaluate left ventricular performance obtained by ICG (supine and orthostatic positions) and TTE was performed by regression analysis using generalized linear mixed effects models.

\begin{tabular}{|c|c|c|c|c|}
\hline \multirow[b]{2}{*}{ Parameter } & Coefficient estimate $(95 \% \mathrm{Cl})$ & $p$ Value & Coefficient estimate $(95 \% \mathrm{Cl})$ & $p$ Value \\
\hline & \multicolumn{2}{|c|}{ ICG in supine } & \multicolumn{2}{|c|}{ ICG in orthostatic } \\
\hline $\mathrm{CO}$ & $-0.01(-0.016 ; 0.004)$ & 0.216 & $0.03(-0.132 ; 0.188)$ & 0.731 \\
\hline $\mathrm{Cl}$ & $-0.02(-0.055 ; 0.022)$ & 0.403 & $0.12(-0.072 ; 0.096)$ & 0.777 \\
\hline PEP & $0.22(-0.125 ; 0.558)$ & 0.214 & $22.65(19.176 ; 26.124)$ & $<0.001$ \\
\hline LVET & $0.14(-0.371 ; 0.651)$ & 0.592 & $-25.62(-33.479 ;-17.757)$ & $<0.001$ \\
\hline STR & $0.00(-0.001 ; 0.001)$ & 0.890 & $0.10(-0.086 ; 0.117)$ & $<0.001$ \\
\hline
\end{tabular}

95\% Cl: 95\% Confidence interval; Cl: cardiac index; CO: cardiac output; ICG: impedance cardiography; LVET: left ventricular ejection time; PEP: pre-ejection period; STR: systolic time ratio; TTE: transthoracic echocardiography.

TTE is the reference category.

greater than $5 \%$ in $\mathrm{HR}$ and $10 \%$ in $\mathrm{BP}$, by minimizing hemodynamic variability, may also have contributed to this good agreement between methods.

The evaluation of the hemodynamic response to postural change from supine to upright position provides useful information on the cardiovascular reflex activity (51). The change from supine to orthostatic position in healthy individuals provokes reduction in thoracic blood volume and stroke volume with subsequent decrease pressure on carotid baroreceptors which then causes a rise in HR and posterior sympathetic vasoconstriction with BP normalization. Associated to the high $\mathrm{HR}$, the systolic time intervals usually decrease $(52,53)$.

Most of the studies were designed to study syncope and therefore differences in orthostatic hemodynamics have not been truly explored among hypertensive patients (54). It was found that age was a major determinant of the response to orthostatism, as those with stable stroke volume and CO were mostly older hypertensive patients eventually related with impaired carotid baroreflex or impaired autonomic balance between arterial and cardiopulmonary baroreflexes (51).

The sample in the present study has many resistant, polymedicated, hypertensive patients with multiple comorbidities, such as diabetes mellitus, which may affect the hemodynamic response to orthostatism. This may contribute to the great dispersion detected for ICG-derived indexes (PEP, LVET, STR) and may explain the poor agreement between ICGderived indexes in orthostatic position and those obtained by TTE, which is performed in supine position.

Echocardiographic measures of LV structure, morphology, and function still continue to be important in the evaluation of heart damage in hypertension (26). However, the significant differences found between ICG-derived indexes in supine/orthostatic position and TTE evaluations indicate that the individual analysis of hypertensive patients with ICG in both positions may add information about the patients' pathophysiological response, improving their hemodynamic characterization and therapeutic optimization.

Future studies in the area of ICG may increase our understanding of the pathophysiology and hemodynamic changes of arterial hypertension, in particular the importance of postural variability, and evidence that early diagnosis and treatment of these hemodynamic characteristics have a positive impact on patient outcomes, reducing morbidity and mortality associated with arterial hypertension.

This study had some limitations. This is a concordance analysis, not strictly a validation study, as the ICG-derived parameters are compared with TTE-derived parameters, which is not the gold standard, but the current clinical standard. It is a post-hoc analysis, based on data obtained for a study whose sample size was intended to reach a different goal. ICG and TTE were performed not simultaneously but within a time lapse of 8 days, a period during which the patients were assumed to remain clinically stable, without any changes of therapy. In order to minimize these weaknesses, a special effort was paid to guarantee the validity of the measurements. Sampling had strict inclusion and exclusion criteria; patients were submitted to the same well-defined evaluation protocols, performed in a controlled clinical environment by experienced professionals. Moreover, evaluations matching variations $>10 \%$ in $\mathrm{BP}$ or variations $>5 \%$ in $\mathrm{HR}$ were not considered.

\section{Conclusions}

There is strong evidence that ICG performed in supine position can accurately evaluate LV performance in hypertensive patients, as ICG-derived systolic time intervals, CO, and CI have good agreement with the parameters obtained by the clinical standard (TTE).

\section{Acknowledgments}

We would like to thank José Rola, Júlio Almeida, and Luís Dias who contributed with their knowledge, expertise to the protocol design, and allowed IMPEDDANS study to be successfully completed in the departments they lead. We also would like to thank the cardiopneumology technicians Helena Fonseca and Paula Ribeiro for their effort and dedication performing ICG exams and Luisa Branco, Júlio Calaça, and Pedro Rio for their performance and availability in the realization and revision of echocardiograms.

\section{Declaration of interest}

The authors report no conflicts of interest. The authors alone are responsible for the content and writing of the paper.

\section{ORCID}

Rodrigo Nazário Leão (D) http://orcid.org/0000-0002-4823-3669

\section{References}

1. Bour J, Kellett J. Impedance cardiography: a rapid and cost-effective screening tool for cardiac disease. Eur J Intern Med. 2008;19 (6):399-405. 
2. Tahvanainen A, Koskela J, Leskinen M, Ilveskoski E, Nordhausen K, Kähönen M, Kööbi T, Mustonen J, Pörsti I. Reduced systemic vascular resistance in healthy volunteers with presyncopal symptoms during a nitrate-stimulated tilt-table test. $\mathrm{Br} \mathrm{J}$ Clin Pharmacol. 2011;71(1):41-51.

3. DeMarzo AP, Calvin JE. A new approach for low-cost noninvasive detection of asymptomatic heart disease at rest. Prev Cardiol. 2007;10(1):9-14.

4. Gielerak G, Piotrowicz E, Krzesinski P, Kowal J, Grzeda M, Piotrowicz R. The effects of cardiac rehabilitation on haemodynamic parameters measured by impedance cardiography in patients with heart failure. Kardiol Pol. 2011;69(4):309-17.

5. Khan FZ, Virdee MS, Hutchinson J, Smith B, Pugh PJ, Read PA, Fynn SP, Dutka DP. Cardiac resynchronization therapy optimization using noninvasive cardiac output measurement. Pacing Clin Electrophysiol. 2011;34(11):1527-36.

6. Balachandran JS, Bakker JP, Rahangdale S, Yim-Yeh S, Mietus JE, Goldberger AL, Malhotra A. Effect of mild, asymptomatic obstructive sleep apnea on daytime heart rate variability and impedance cardiography measurements. Am J Cardiol. 2012;109 (1):140-45.

7. Tomsin K, Mesens T, Molenberghs G, Gyselaers W. Venous pulse transit time in normal pregnancy and preeclampsia. Reprod Sci. 2012;19(4):431-36.

8. Ferrario CM, Flack JM, Strobeck JE, Smits G, Peters C. Individualizing hypertension treatment with impedance cardiography: a meta-analysis of published trials. Ther Adv Cardiovasc Dis. 2010;4(1):5-16.

9. Nazario Leao R, Marques Da Silva P. Diastolic dysfunction in hypertension. Hipertens Riesgo Vasc. 2017;34(3):128-139.

10. Sramek BBTJ, Hojerov M. Cervenka V Normohemodynamic goal -oriented antihypertensive therapy improves the outcome. Am J Hypertens. 1996;9:141A.

11. Taler SJ, Textor SC, Augustine JE. Resistant hypertension: comparing hemodynamic management to specialist care. Hypertension. 2002;39(5):982-88.

12. Sharman DL, Gomes CP, Rutherford JP. Improvement in blood pressure control with impedance cardiography-guided pharmacologic decision making. Congest Heart Fail. 2004;10(1):54-58.

13. Smith RD, Levy P, Ferrario CM. Consideration of noninvasive hemodynamic monitoring to target reduction of blood pressure levels study G. Value of noninvasive hemodynamics to achieve blood pressure control in hypertensive subjects. Hypertension. 2006;47(4):771-77.

14. Krzesinski P, Gielerak G, Kowal J, Piotrowicz K. Usefulness of impedance cardiography in optimisation of antihypertensive treatment in patients with metabolic syndrome: a randomised prospective clinical trial. Kardiol Pol. 2012;70(6):599-607.

15. Krzesinski P, Gielerak G, Stanczyk A, Piotrowicz K, Skrobowski A. Who benefits more from hemodynamically guided hypotensive therapy? The experience from two randomized, prospective and controlled trials. Ther Adv Cardiovasc Dis. 2016;10(1):21-29.

16. Messerli FH, Rimoldi SF, Bangalore S. The transition from hypertension to heart failure: contemporary update. JACC Heart Fail. 2017;5(8):543-551.

17. DeMarzo AP, Kelly RF, Calvin JE. Impedance cardiography: a comparison of cardiac output vs waveform analysis for assessing left ventricular systolic dysfunction. Prog Cardiovasc Nurs. 2007;22(3):145-51.

18. Thompson B, Drazner MH, Dries DL, Yancy CW. Systolic time ratio by impedance cardiography to distinguish preserved vs impaired left ventricular systolic function in heart failure. Congest Heart Fail. 2008;14(5):261-65.

19. Talley RC, Meyer JF, McNay JL. Evaluation of the pre-ejection period as an estimate of myocardial contractility in dogs. Am J Cardiol. 1971;27(4):384-91.

20. Martin CE, Shaver JA, Thompson ME, Reddy PS, Leonard JJ. Direct correlation of external systolic time intervals with internal indices of left ventricular function in man. Circulation. 1971;44 (3):419-31.
21. Sant Anna JRM. Valorização do período de pré-ejeção na dinâmica cardiovascular. Rev Bras Marcapasso E Arritmia. 1971;7(1):5-12.

22. Weber T, Auer J, O’Rourke MF, Punzengruber C, Kvas E, Eber B. Prolonged mechanical systole and increased arterial wave reflections in diastolic dysfunction. Heart. 2006;92(11):1616-22.

23. Kaszuba E, Scheel S, Odeberg H, Halling A. Comparing impedance cardiography and echocardiography in the assessment of reduced left ventricular systolic function. BMC Res Notes. 2013;6:114.

24. Carvalho P, Paiva RP, Couceiro R, Henriques J, Antunes M, Quintal I, Muehlsteff J, Aubert X. Comparison of systolic time interval measurement modalities for portable devices. Conf Proc IEEE Eng Med Biol Soc. 2010;2010:606-09.

25. Wang DJ, Gottlieb SS. Impedance cardiography: more questions than answers. Curr Cardiol Rep. 2006;8(3):180-86.

26. Mancia G, Fagard R, Narkiewicz K, Redón J, Zanchetti A, Böhm M, Christiaens T, Cifkova R, De Backer G, Dominiczak A, et al. ESH/ESC guidelines for the management of arterial hypertension: the task force for the management of arterial hypertension of the European Society of Hypertension (ESH) and of the European Society of Cardiology (ESC). Eur Heart J. 2013;34(28):2159-219.

27. New York Heart Association. Criteria C. Nomenclature and criteria for diagnosis of diseases of the heart and great vessels. 7th ed. Boston: Little, Brown and Company; 1973.

28. Galiuto L. The EAE textbook of echocardiography. Oxford: Oxford University Press; 2011.

29. Reant P, Dijos M, Donal E, Mignot A, Ritter P, Bordachar P, Dos Santos P, Leclercq C, Roudaut R, Habib G, Lafitte S. Systolic time intervals as simple echocardiographic parameters of left ventricular systolic performance: correlation with ejection fraction and longitudinal two-dimensional strain. Eur J Echocardiogr. 2010;11 (10):834-44.

30. Hinkle DE, Wiersma W, Jurs SG. Applied statistics for the behavioral sciences 5th ed. Boston, MA: Houghton Mifflin; [London: Hi Marketing] [distributor]; 2003.

31. Fellahi JL, Caille V, Charron C, Deschamps-Berger PH, VieillardBaron A. Noninvasive assessment of cardiac index in healthy volunteers: a comparison between thoracic impedance cardiography and Doppler echocardiography. Anesth Analg. 2009;108 (5):1553-59.

32. Dazzani F, Micati M, Caraceni P, Drago GM, Domenicali M, Pacilli P, Tomassetti V, Gelonesi E, Trevisani F, Bernardi M. Transthoracic electrical bioimpedance: a non-invasive technique for the evaluation of the haemodynamic alterations in patients with liver cirrhosis. Dig Liver Dis. 2005;37(10):786-92.

33. Cybulski G, Michalak E, Kozluk E, Piatkowska A, Niewiadomski W. Stroke volume and systolic time intervals: beat-to-beat comparison between echocardiography and ambulatory impedance cardiography in supine and tilted positions. Med Biol Eng Comput. 2004;42(5):707-11.

34. Antonicelli R, Savonitto S, Gambini C, Tomassini PF, Sardina M, Paciaroni E. Impedance cardiography for repeated determination of stroke volume in elderly hypertensives: correlation with pulsed Doppler echocardiography. Angiology. 1991;42(8):648-53.

35. Aust PE, Belz GG, Belz G, Koch W. Comparison of impedance cardiography and echocardiography for measurement of stroke volume. Eur J Clin Pharmacol. 1982;23(6):475-77.

36. Fuller HD. The validity of cardiac output measurement by thoracic impedance: a meta-analysis. Clin Invest Med. 1992;15(2):10312.

37. Raaijmakers E, Faes TJ, Scholten RJ, Goovaerts HG, Heethaar RM. A meta-analysis of three decades of validating thoracic impedance cardiography. Crit Care Med. 1999;27(6):1203-13.

38. Peyton PJ, Chong SW. Minimally invasive measurement of cardiac output during surgery and critical care: a meta-analysis of accuracy and precision. Anesthesiology. 2010;113(5):1220-35.

39. Fellahi JL, Fischer MO. Electrical bioimpedance cardiography: an old technology with new hopes for the future. J Cardiothorac Vasc Anesth. 2014;28(3):755-60. 
40. Woltjer HH, Bogaard HJ, Van Der Spoel HI, De Vries PM. The influence of weight on stroke volume determination by means of impedance cardiography in cardiac surgery patients. Intensive Care Med. 1996;22(8):766-71.

41. Drazner MH, Thompson B, Rosenberg PB, Kaiser PA, Boehrer JD, Baldwin BJ, Dries DL, Yancy CW. Comparison of impedance cardiography with invasive hemodynamic measurements in patients with heart failure secondary to ischemic or nonischemic cardiomyopathy. Am J Cardiol. 2002;89(8):993-95.

42. Kamath SA, Drazner MH, Tasissa G, Rogers JG, Stevenson LW, Yancy CW. Correlation of impedance cardiography with invasive hemodynamic measurements in patients with advanced heart failure: the BioImpedance CardioGraphy (BIG) substudy of the Evaluation Study of Congestive Heart Failure and Pulmonary Artery Catheterization Effectiveness (ESCAPE) Trial. Am Heart J. 2009;158(2):217-23.

43. Lorne E, Mahjoub Y, Diouf M, Sleghem J, Buchalet C, Guinot PG, Petiot S, Kessavane A, Dehedin B, Dupont H. Accuracy of impedance cardiography for evaluating trends in cardiac output: a comparison with oesophageal Doppler. Br J Anaesth. 2014;113(4):596-602.

44. Bernstein DP, Lemmens HJ, Brodsky JB. Limitations of impedance cardiography. Obes Surg. 2005;15(5):659-60.

45. Critchley LA, Calcroft RM, Tan PY, Kew J, Critchley JA. The effect of lung injury and excessive lung fluid, on impedance cardiac output measurements, in the critically ill. Intensive Care Med. 2000;26(6):679-85.

46. Balestra B, Malacrida R, Leonardi L, Suter P, Marone C. Esophageal electrodes allow precise assessment of cardiac output by bioimpedance. Crit Care Med. 1992;20(1):62-67.
47. Faini A, Omboni S, Tifrea M, Bubenek S, Lazar O, Parati G. Cardiac index assessment: validation of a new non-invasive very low current thoracic bioimpedance device by thermodilution. Blood Press. 2014;23(2):102-08.

48. Treister N, Wagner K, Jansen PR. Reproducibility of impedance cardiography parameters in outpatients with clinically stable coronary artery disease. Am J Hypertens. 2005;18(2 Pt 2):44S$50 \mathrm{~S}$.

49. Greenberg BH, Hermann DD, Pranulis MF, Lazio L, Cloutier D. Reproducibility of impedance cardiography hemodynamic measures in clinically stable heart failure patients. Congest Heart Fail. 2000;6(2):74-80.

50. Verhoeve PE, Cadwell CA, Tsadok S. Reproducibility of noninvasive bioimpedance measurements of cardiac function. J Card Fail. 1998;4(Suppl 1):53.

51. Bettencourt MJ, Pinto BG, De Oliveira EI, Silva-Carvalho L. Two anomalous cardiovascular responses to active standing in essential hypertension. Rev Port Cardiol. 2008;27(5):613-21.

52. Smith JJ, Porth CM, Erickson M. Hemodynamic response to the upright posture. J Clin Pharmacol. 1994;34(5):375-86.

53. Smith JJ, Muzi M, Barney JA, Ceschi J, Hayes J, Ebert TJ. Impedance-derived cardiac indices in supine and upright exercise. Ann Biomed Eng. 1989;17(5):507-15.

54. Tahvanainen AM, Tikkakoski AJ, Koskela JK, Nordhausen K, Viitala JM, Leskinen MH, Kähönen MA, Kööbi T, Uitto MT, Viik J. et al. The type of the functional cardiovascular response to upright posture is associated with arterial stiffness: a crosssectional study in 470 volunteers. BMC Cardiovasc Disord. 2016;16:101. 